Transplantation 2013; 19:1413-1414.

3. Ison MG, Nalesnik MA. An Update on Donor-Derived Disease Transmission in Organ Transplantation. American Journal of Transplantation 2011; 11: 1123-1130

4. Tan FLS, Loh DLSK, Prabhakaran K. Dengue haemorrhagic fever after living donor renal transplantation. Nephrol Dial Transplant. 2005; 20: 447-448.

5. Gupta RK, Gupta G, Chorasiya VK, Bag P, Shandil R, Bhatia V, Wadhawan M, Vij V, Kumar A. Dengue Virus Transmission from Living Donor to Recipient in Liver Transplantation: A Case Report. Journal of Clinical and Experimental Hepatology. 2016; 6:1: 59-61

\section{Carcinoma Gall Bladder with Cardiac Metastasis Presenting as Liver Abscess: An Unusual Case}

Carcinoma Gall Bladder (CaGB) is the most common biliary tract malignancy with geographic and ethnic disparities. It is one of the most common malignancies in north India, particularly in females. It has a vague presentation and mostly is detected in advanced stage. We here in report an interesting case which initially presented as liver abscess and latter diagnosed as gall bladder cancer with cardiac metastasis.

\section{Case Report}

A 55 year old gentleman presented to us with persistent fever and pain abdomen for the last one and half months. He was evaluated elsewhere with USG and CECT of the abdomen. A diagnosis of liver abscess was made and he was treated with antibiotics and percutaneous catheter drainage of the abscess cavity. The catheter was removed after three days. He continued to be symptomatic and was given a long course of antibiotics. At presentation to our hospital he was febrile, septic but hemodynamically stable, anicteric with no lymphadenopathy. Abdominal examination was unremarkable. Blood investigations revealed a raised total count of $21900 \mathrm{c} / \mathrm{mm}^{3}$, normal bilirubin and liver enzymes with raised Serum Alkaline Phosphatase. He was reassessed with CECT which showed a large partly exophytic relatively well defined necrotic heterogenously enhancing mass lesion measuring approx. $9.6 \times 10.3 \times 9.9$ in segment IVA, IVB, V and VIII of liver with gall bladder embedded within the mass lesion (Figure 1A and 1B), with heterogenously hypodense lesion measuring $2.7 \times 2.3 \mathrm{~cm}$ in right ventricle (Figure 1C and 1D), suggestive of gall bladder cancer with intracardiac metastasis. Tumour markers were evaluated which showed CEA-23.19, CA 19-9 100.5. ECHO showed a $2.5 \times 4.6 \mathrm{~mm}$ homogenous mass in the Right ventricular apex with no free mobility. FNAC from the gall bladder mass confirmed the diagnosis of gall bladder cancer. In view of his poor performance status he

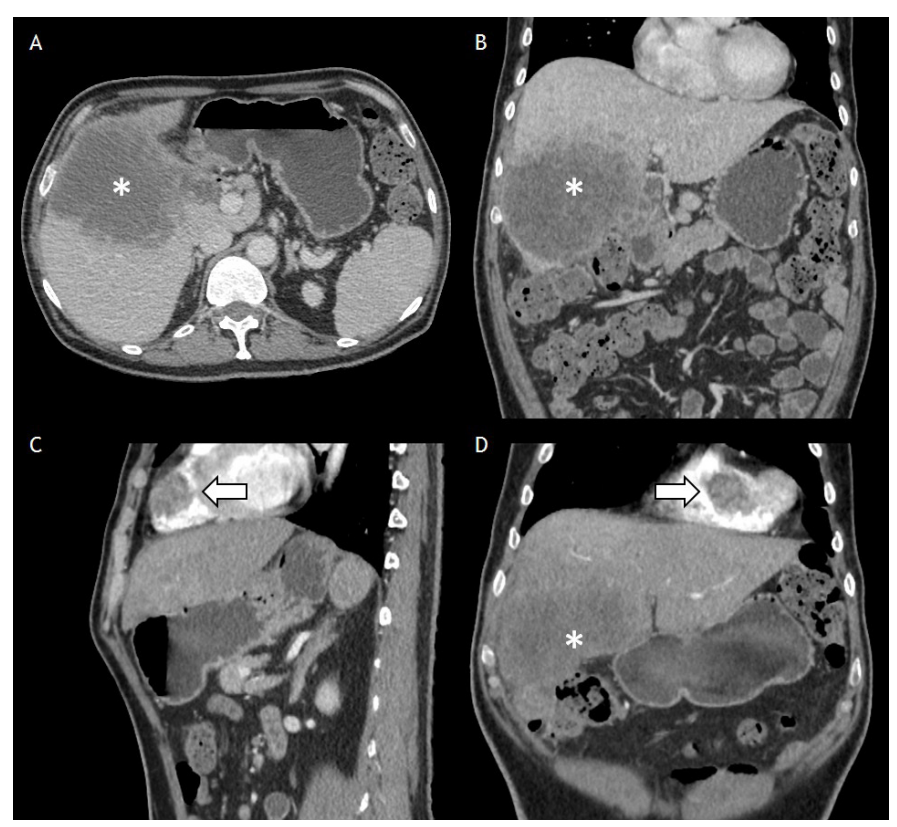

Figure 1(A \& B): Thin venous phase image showing relatively well defined necrotic heterogenously enhancing mass lesion measuring approx. 9.6 x 10.3 $x 9.9$ in segment IVA, IVB, V and VIII of liver with gall bladder embedded within the mass lesion (asterix mark). (C \& D): Thin cut arterial phase imaging showing heterogenously hypodense lesion measuring $2.7 \times 2.3 \mathrm{~cm}$ in right ventricle- Intracardiac metastasis (arrow mark). 
was planned for best supportive care. He succumbed to his disease within 2 months of presentation.

\section{Discussion}

Metastatic spread from primary $\mathrm{CaGB}$ is usually to the liver and the paraaortic lymph nodes. The modes of dissemination in gallbladder carcinoma are direct, lymphatic, vascular, neural, intraperitoneal, and intraductal. There have been reports of $\mathrm{CaGB}$ metastasising to unusual sites like bone ${ }^{1}$, brain ${ }^{1}$, ovary ${ }^{2}$, spine $^{3}$, cheek ${ }^{4}$ and breast ${ }^{5}$. A handful cases with cardiac metastasis have also been reported. ${ }^{6-9}$ Gall bladder cancer can very rarely mimic a liver abscess, which may come to light only after drainage or at the time of surgery.

We herein report an extremely uncommon combination of two rare presentations of gall bladder cancer in a patient i.e. gall bladder cancer presenting as liver abscess and subsequently developing cardiac metastasis. Cardiac metastasis may develop via lymphatic, haematogenous or direct spread. But myocardial involvement like in our case is usually through haematogenous spread. Most cardiac metastasis are asymptomatic and are detected incidentally or at the time of autopsy. ${ }^{7-10}$ Autopsy studies of metastatic neoplasms of the heart have reported gallbladder carcinoma as a rare primary site. ${ }^{10}$

Only one case has been reported of a gall bladder cancer presenting with pyrexia of unknown origin and misdiagnosed as liver abscess on $\mathrm{CT}$, as in our case and later found to have carcinoma only at surgery. ${ }^{11}$

\section{Conclusion}

The case illustrates an unusual presentation of gallbladder carcinoma with a combination of a rare demonstration of it as liver abscess with cardiac metastasis. At the same time, it highlights the highly aggressive nature of gallbladder carcinoma, progressing from asymptomatic to death within two months. Thus an early diagnosis is essential in cases of $\mathrm{CaGB}$ and improved imaging modalities and its interpretation supplemented with accurate diag $\neg$ nostic markers will potentially help outcomes.
SAPHALTA BAGHMAR ${ }^{1}$ ROMMEL SANDHYAV ${ }^{2}$ ASIT ARORA $^{2}$ ANKUR ARORA ${ }^{3}$

\author{
${ }^{1}$ Department of Medical Oncology, \\ ${ }^{2}$ Department of Hepatopancreatobiliary surgery, \\ ILBS, Vasant Kunj, New Delhi 110070, India \\ ${ }^{3}$ Consultant Radiologist, Worthing Hospital, \\ Western Sussex NHS Trust \\ Corresponding Author: Dr Asit Arora \\ Email:drasitarora@yahoo.com
}

\section{References}

1. Aung Z. Win, Carina M. Aparici, Rare case of gallbladder cancer presenting with metastasis to bone and brain in an African American male discovered by F18-FDG PET/CT, J Biomed Graph Comput. 2013;3(2) 24-29

2. Kumar Y, Chahal A, Garg M, Bhutani A. Occult gallbladder carcinoma presenting as a primary ovarian tumor in two women: two case reports and a review of the literature. $\mathrm{J}$ Med Case Reports. 2010 Jun 30;4(1):202.

3. Joshi MK, Joshi R, Chadha M, Alam SE, Varshneya H, Kumar S. Gall bladder carcinoma presenting with spinal metastasis: a rare phenomenon. Indian J Palliat Care. 2013 May;19(2):113-5.

4. Marin H, Bouras AF, Patenôtre P, Boleslawski E, Zerbib P, Pruvot FR, et al. Cheek metastasis from gallbladder adenocarcinoma. J Visc Surg. 2013 Jun;150(3):225-6.

5. Shukla P, Roy S, Tiwari V, Mohanti BK. Unusual presentation of metastatic gall bladder cancer. J Cancer Res Ther. 2014 Jun;10(2):397-8.

6. Gunjiganvi M, Singh KK, Harsha HS, Bipin T. Cardiac metastasis from gallbladder carcinoma. Int $\mathrm{J}$ Surg Case Rep. 2013 Oct 22;4(12):1156-8.

7. Suganuma M, Marugami Y, Sakurai Y, Ochiai M, Hasegawa S, Imazu H, et al. Cardiac metastasis from squamous cell carcinoma of gallbladder. J Gastroenterol. 1997 Dec;32(6):852-6.

8. Sugezawa A, Hiraoka H, lizuka Y, Nishimura K, Kishi H, Furuse $\mathrm{T}$, et al. [Heart metastasis of gallbladder cancer-a case report]. Gan No Rinsho Jpn J Cancer Clin. 1987 Apr;33(4):406-10.

9. Inoue T, Shiraki K, Fuke H, Yamanaka Y, Miyashita K, Ito $\mathrm{K}$, et al. Cardiac metastases of gallbladder carcinoma. World J Gastroenterol. 2005 Apr 7;11(13):2048-9. 
10. Hanfling SM. Metastatic cancer to the heart. Review of the literature and report of 127 cases. Circulation. 1960 Sep;22:474-83.

11. Singla SL, Garg P, Tahlan RN. Gall bladder carcinoma presenting as liver abscess. Indian J Gastroenterol Off J Indian Soc Gastroenterol. 1998 Apr;17(2):68.

\section{Reversal of Vitiligo with Cure from Hepatitis-C Infection}

Hepatitis-C infection has been associated with various dermatological extra-hepatic manifestations. ${ }^{1}$ We report a patient of chronic hepatitis-C infection with vitiligo which improved on successful treatment of hepatitis-C infection.

\section{Case Report}

65-year-old lady was diagnosed with hepatitis-C related cirrhosis when she presented with an oesophageal variceal bleed for which she had undergone endoscopic variceal ligation. She gave history of a caesarean section 35 years ago with no history of blood transfusion, intravenous drug abuse, tattooing or any history suggestive of autoimmune disease in herself or her family. She gave history of skin de-pigmentation which started 5 years back on her face and gradually became generalised over next 2 years (Figure 1). She had generalised, well-defined, hypopigmented patches, suggestive of vitiligo and an enlarged liver palpable $2 \mathrm{~cm}$ below right costal margin. Investigations revealed WB Ccount:4300/ $\mathrm{mm}^{3}$, hemoglobin:11.8 g/dl, platelet count:88000/ $/ \mathrm{mm}^{3}$, total bilirubin:0.9 mg/dl, AST:250 IU/L,ALT:19 IU/L, alkaline phosphatase:180 IU/L (normal range 44-147 IU/L) and serum creatinine: $0.7 \mathrm{mg} / \mathrm{dl}$. The $\mathrm{HbA} 1 \mathrm{C}$ was $4.6 \%$, TSH:1.48 $\mu \mathrm{IU} / \mathrm{ml}$ (normal range 0.30-5.5 $\mu \mathrm{IU} / \mathrm{ml}$ ), ANA titre- 1:160, HBsAg and HIV were non-reactive. Anti-HCV was reactive with Hepatitis C viral load of 5,293,804 IU/ml(COBAS TaqMan technique) and genotype 3a. Ultrasonography abdomen was suggestive of cirrhosis with portal hypertension and ascites. She was started on PEG-interferon $\alpha$-2a 180 mcg subcutaneously weekly with ribavirin $800 \mathrm{mg} /$ day. She had not achieved a rapid virological response. After 2 months of initiating treatment, sofosbuvir was added to the treatment regime since it had become available in India. She received the three drugs-PEG-interferon, ribavarin and sofosbuvir for 6 months. Erythropoietin was administered as she developed ribavirin-induced anaemia, with lowest recorded haemoglobin being $8 \mathrm{~g} / \mathrm{dL}$. She achieved a rapid virological response at 1 month after initiation of the triple drug therapy. The vitiligo started improving around 3 months after initiation of this therapy, with reappearance of pigmented areas starting with her face and then extended to rest of her body (Figure 2). She achieved end of treatment response and sustained virological response at 12 and 24 weeks respectively post completion of therapy.

\section{Discussion}

Prevalence of HCV infection in India is $1.3 \%{ }^{2}$ Dermatological manifestations of HCV maybe a result of direct action of viral particles on keratinocytes, lymphocytes, antigen presenting cells and blood

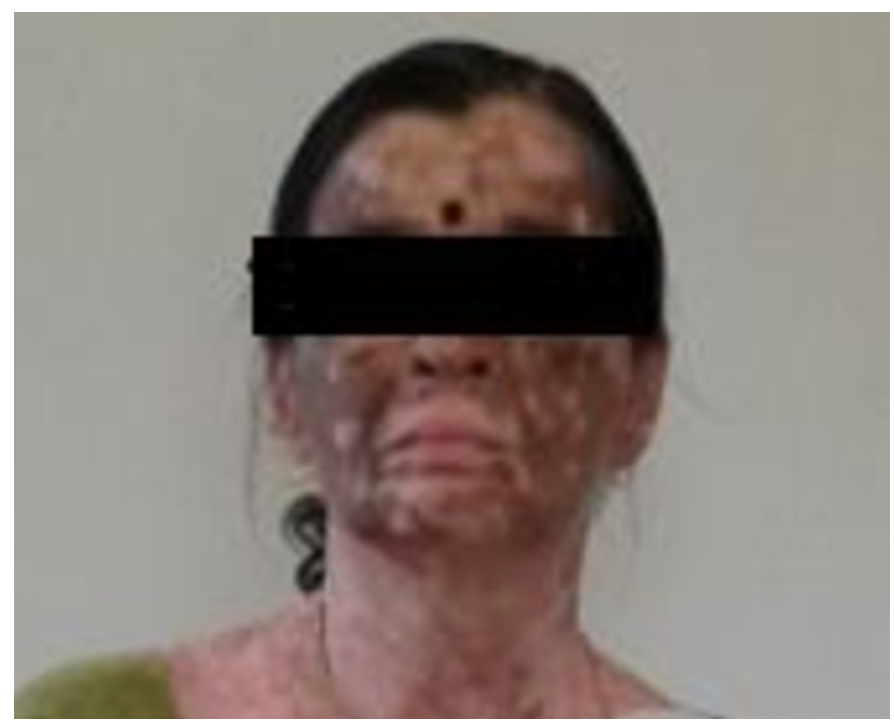

Figure 1: Vitiligo areas at the time of presentation. 